\section{DISCUSSION}

Aspergillus is a rare cause of endocarditis. ${ }^{1-3}$ The prognosis for $\mathrm{AE}$ is poor, with only a third of reported patients surviving the acute episode, in part because of immunocompromise, delay in diagnosis, systemic dissemination, and multiple embolic episodes. ${ }^{1}$ Only 5 cases of infective endocarditis caused by the species A terreus have been reported. ${ }^{1}$ To the best of our knowledge, this is the first reported case of prosthetic valve endocarditis caused by $A$ terreus.

Early and accurate diagnosis is the most critical step in the treatment of $\mathrm{AE}$ and requires a high index of suspicion. The occurrence of peripheral emboli is the most common presenting feature and in a culture-negative infective endocarditis setting should raise the suspicion of $\mathrm{AE} .{ }^{1,3,4}$ Echocardiography reveals large vegetations on the valve(s), but blood cultures are almost always negative..$^{1-3}$ Ultimately, the diagnosis of Aspergillus requires histology and tissue culture confirmation from vegetations or emboli. Determining species is critical, because A terreus species is almost always resistant to amphotericin $\mathrm{B}$, and voriconazole is the antifungal drug of choice, often in combination with an echinocandin. ${ }^{1}$

Mortality is extremely high among those who receive antifungal therapy alone. ${ }^{1-3}$ Successful treatment of $\mathrm{AE}$ requires combination of appropriate antifungal therapy and radical surgical debridement. Despite adequate combined antifungal and radical surgical treatment, recurrence after $\mathrm{AE}$ as high as $40 \%$ has been reported, adding to the dismal prognosis. ${ }^{1-3}$ As a consequence, we now recommend antifungal suppression for life.
Our patient was not immunosuppressed. El-Hamamsy and colleagues reported a cluster of 3 immunocompetent cases of $\mathrm{AE}$ after aortic valve replacement in which the source could not be identified. ${ }^{5}$ They suggested seeding by airborne spores as probable cause, emphasizing importance of operating room sterility and use of highefficiency particulate air filters.

In conclusion, $\mathrm{AE}$ is a rare, often fatal infection requiring a high index of suspicion, multidisciplinary team approach for expeditious and accurate diagnosis, and appropriate antifungal and surgical treatment. Diagnosis requires histology and culture. Species diagnosis of AE is critical, because A terreus is resistant to amphotericin B. Immediate surgical intervention with radical debridement and lifelong antifungal therapy remains the best hope of improved prognosis.

\section{References}

1. Kalokhe AS, Rouphael N, El Chami MF, Workowski KA, Ganesh G, Jacob JT Aspergillus endocarditis: a review of the literature. Int J Infect Dis. 2010;14 e1040-7.

2. Gumbo T, Taege AJ, Mahorter S, McHenry MC, Lytle BW, Cosgrove DM, et al. Aspergillus valve endocarditis in patients without prior cardiac surgery. Medicine (Baltimore). 2000;79:261-8.

3. El-Hamamsy I, Dürrleman N, Stevens LM, Perrault LP, Carrier M Aspergillus endocarditis after cardiac surgery. Ann Thorac Surg. 2005; 80:359-64.

4. Ryu KM, Seo PW, Kim SH, Park S, Ryu JW. Surgical treatment of native valve Aspergillus endocarditis and fungemic vascular complications. J Korean Med Sci. 2009;24:170-2.

5. El-Hamamsy I, Dürrleman N, Stevens LM, Cartier R, Pellerin M, Perrault LP, et al A cluster of cases of Aspergillus endocarditis after cardiac surgery. Ann Thorac Surg. 2004;77:2184-6.

\title{
Familial Ebstein's anomaly, left ventricular noncompaction, and ventricular septal defect associated with an $\mathrm{MYH7}$ mutation
}

\author{
Keiichi Hirono, MD, ${ }^{a}$ Yukiko Hata, PhD, ${ }^{b}$ Keijirou Ibuki, MD, ${ }^{a}$ and Naoki Yoshimura, MD, ${ }^{\mathrm{c}}$ Toyama, Japan
}

Left ventricular noncompaction (LVNC) is a rare cardiomyopathy characterized by prominent trabeculations with deep intertrabecular recesses. ${ }^{1}$ Ebstein's anomaly is a rare congenital heart defect, and familial cases are

\footnotetext{
From the Departments of Pediatrics, ${ }^{\mathrm{a}}$ Legal Medicine, ${ }^{\mathrm{b}}$ and 1st Surgery, ${ }^{\mathrm{c}}$ Graduate School of Medicine, University of Toyama, Toyama, Japan.

Disclosures: Authors have nothing to disclose with regard to commercial support. Received for publication Aug 11, 2014; accepted for publication Aug 20, 2014.

Address for reprints: Keiichi Hirono, MD, Department of Pediatrics, Graduate School of Medicine, University of Toyama, 2630 Sugitani, Toyama City, Toyama, 9300194 Japan (E-mail: khirono1973@gmail.com).

J Thorac Cardiovasc Surg 2014;148:e223-6

$0022-5223 / \$ 36.00$

Copyright (c) 2014 by The American Association for Thoracic Surgery

http://dx.doi.org/10.1016/j.jtcvs.2014.08.049
}

uncommon. ${ }^{2,3}$ These genetic causes have not been fully elucidated.

We describe clinical and molecular investigations of a rare case of familial Ebstein's anomaly, LVNC, and ventricular septal defect (VSD) in association with a likely pathogenic novel mutation of the MYH7 (c.1085T $>$ G, p.Met362Arg) gene. The sister and brother have been diagnosed with $\mathrm{Eb}$ stein's anomaly, LVNC, and VSD, and novel mutation was identified in the sister, brother, and father.

\section{CLINICAL SUMMARY}

Case 1 (the Proband)

The patient was a full-term infant with a birth weight of $3.42 \mathrm{~kg}$ and Apgar scores of 9 and 10 at 1 and 5 

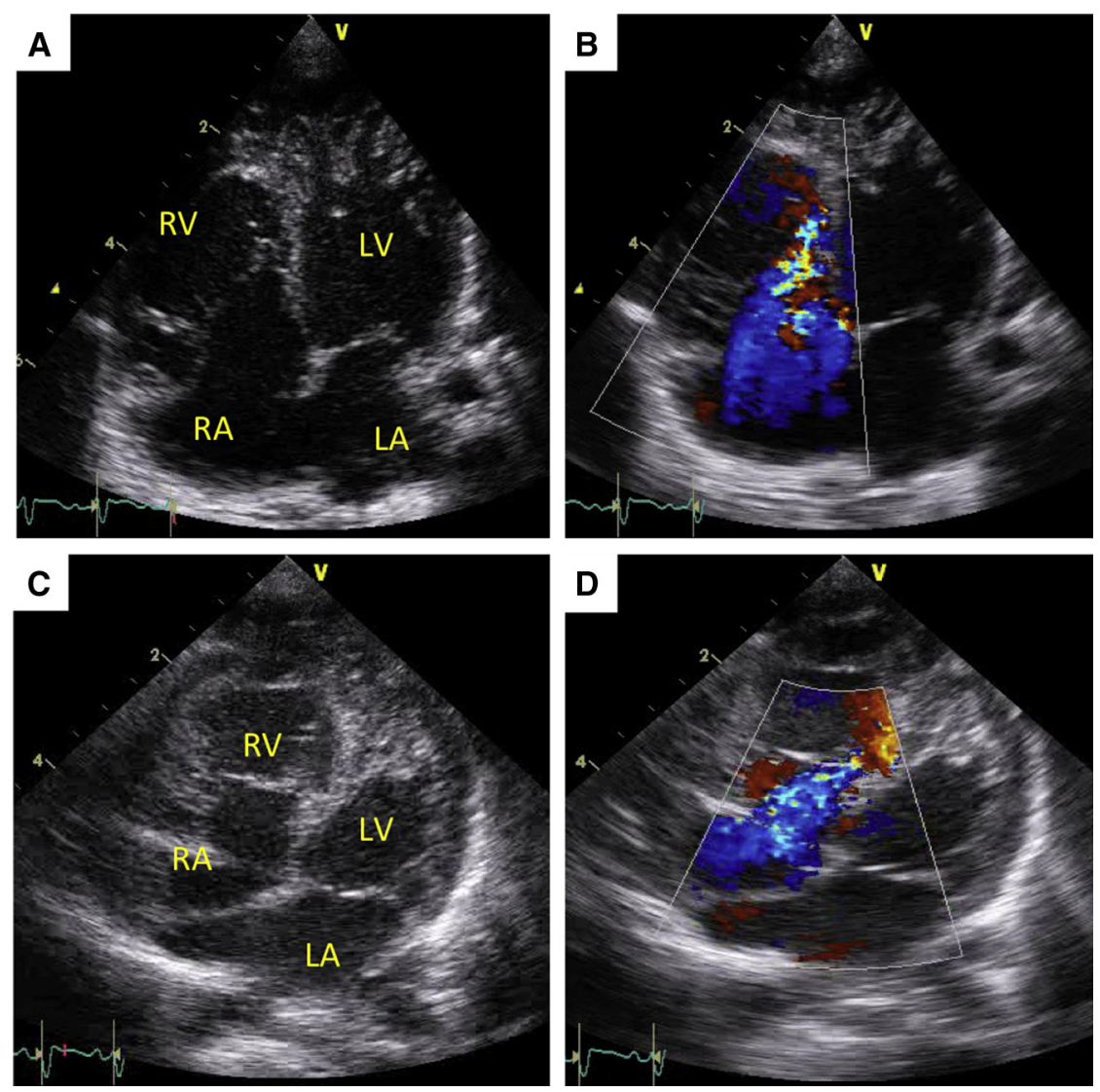

FIGURE 1. Echocardiographic images of case 1 ( $A$ and $B$ ) and case 2 (C and D). In case 1, apical 4-chamber view shows the characteristic left ventricular myocardium without (A) and with (B) color flow visible in intertrabecular recesses on color Doppler images. In case 2, apical 4-chamber view shows the characteristic left ventricular myocardium without (C) and with (D) color flow visible in intertrabecular recesses on color Doppler images. $L A$, Left atrium; $L V$, left ventricle; $R A$, right atrium; $R V$, right ventricle.

minutes, respectively. At 4 days of age, a precordial systolic murmur was audible, and the baby was referred to the Graduate School of Medicine. Echocardiography revealed mild apical displacement of the tricuspid valve, indicating mild Ebstein's anomaly with moderate tricuspid valve insufficiency and right heart enlargement, atrial septal defect, membranous VSD, and LVNC (Figure 1). Criteria used for the echocardiographic diagnosis of LVNC were the presence of prominent or numerous left ventricular trabeculations over the 1-wall segment of the left ventricle, 2-layered appearance of the myocardium with an increased noncompacted to compacted ratio (suggested as $>2.0$ ), and multiple deep intertrabecular recesses communicating with the ventricular cavity, as demonstrated by color Doppler imaging. ${ }^{1,4}$ At 11 days of age, the baby was symptomatic, feeding poorly, pale, and poorly perfused, with tachypnea and tachycardia. She underwent repair of the atrial septal defect and VSD at the age of 16 days. However, her heart failure symptoms did not improve. Coarctation of the aorta was identified by differences in blood pressures between the upper limb and the lower limb, and arch repair was performed at the age of 1 month.

At 3 years of follow-up, the patient is doing well, with normal growth and development, but she continues to have echocardiographic features of LVNC with abnormal myocardial relaxation (Figure 1).

\section{Case 2 (Her Brother)}

Fetal echocardiography at 26 weeks revealed that the fetus also had mild Ebstein's anomaly and VSD. The patient was born as a full-term male infant with a birth weight of $3.42 \mathrm{~kg}$ and Apgar scores of 8 and 8 at 1 and 5 minutes, respectively. At 5 days of age, the baby became gradually symptomatic with tachypnea and tachycardia and was admitted to our hospital. Echocardiography revealed mild Ebstein's anomaly, VSD, and LVNC as identified in his sister (Figure 1). He underwent VSD repair at the age of 1 month.

He is now aged 1 year and in good health. His cardiac function has steadily improved to reach a left ventricular ejection fraction of approximately $60 \%$, but he continues to have echocardiographic features of LVNC with abnormal myocardial relaxation (Figure 1). 

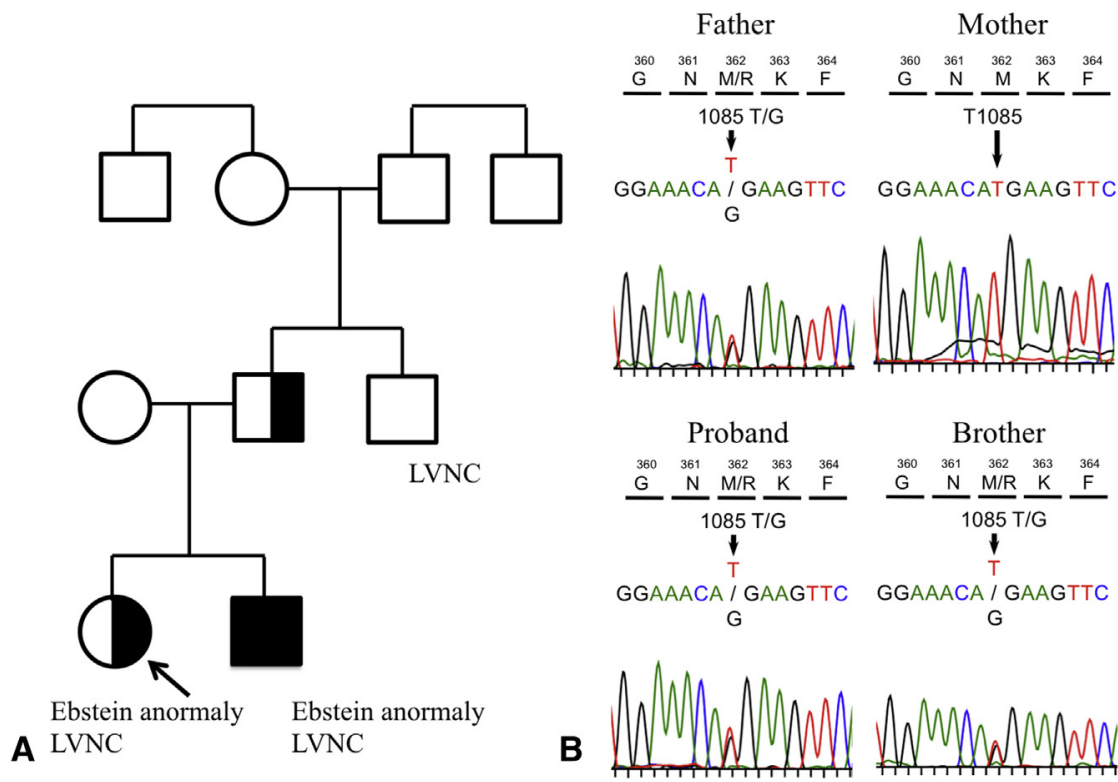

FIGURE 2. The pedigree of the family with Ebstein's anomaly and LVNC (A) and genetic analysis (B). A, The proband is indicated by the arrow. Solid symbols indicate individuals with Ebstein's anomaly and LVNC; half solid symbols indicate individuals with LVNC; open symbols indicate unaffected individuals. B, Sequence analysis of $M Y H 7$ reveals a change of methionine to arginine at position 362 in the proband, his sister, and his father. The variant was considered likely pathogenic because of the following criteria: (1) presence in all affected members of the family; (2) absence from 400 unrelated chromosomes of the control subjects; (3) absence in the public databases, dbSNP database (http://www.ncbi.nlm.nih.gov/projects/SNP/) and National Heart, Lung, and Blood Institute GO Exome Sequencing Project (http://evs.gs.washington.edu/EVS/); (4) affecting evolutionary conserved residues (nucleotide and amino acid); and (5) predicted deleterious/pathogenic by multiple in silico algorithms (Polyphen2, PANTHER, Align GVGD, SHIFT, and Mutation Taster). No mutations were identified in the TAZ, LMNA/C, MYBPC3, TNNT2, TNNI3, ACTC, and TPM1 genes. $L V N C$, Left ventricular noncompaction.

At this time, we were able to obtain some additional history from the parents. They have no limited exercise tolerance and do not have dyspnea on exertion. The echocardiogram revealed the father also has LVNC.

\section{MOLECULAR STUDIES}

A novel $M Y H 7$ heterozygous missense variant (NM_000257.2; c.1085T >G, p.Met362Arg) was identified in the proband and his sister, and was inherited from their father (Figure 2).

\section{DISCUSSION}

Most cases of Ebstein's anomaly are sporadic and familial cases are rare, but various associated cardiovascular abnormalities have been reported, including LVNC or hypertrabeculation of the left ventricle and VSD. ${ }^{2,3}$

The $M Y H 7$ gene, localized on chromosome $14 \mathrm{p} 12$, is composed of 41 exons and encodes the $\beta$-myosin heavy chain, expressed in cardiac muscle. Mutations in $\mathrm{MYH7}$ have been reported in individuals with Ebstein's anomaly, ${ }^{5}$ especially those who also had LVNC. The Met362Arg amino acid change in MYH7 is located in the head region that contains all the necessary elements to generate movement of actin relative to the myosin during adenosine triphosphate hydrolysis, suggesting that the MYH7 mutation is likely to diminish the force generation of cardiac myocytes. The Met362Arg variant has not been reported in cases of familial Ebstein's anomaly. The family described supports autosomal dominant inheritance of Ebstein's anomaly, as suggested by previous reports. ${ }^{2,3}$

\section{CONCLUSIONS}

This is the first report of familial Ebstein's anomaly associated with the Met362Arg mutation of the $M Y H 7$ gene. Our cases indicate that the evaluation of cardiac function, the presence of additional congenital malformations and family history, and the identification of the molecular defect within the family are likely to be important before and after cardiac surgery.

\section{References}

1. Ichida F. Left ventricular noncompaction. Circ J. 2009;73:19-26.

2. Bagur RH, Lederlin M, Montaudon M, Latrabew V, Corneloup O, Iriart X, et al Images in cardiovascular medicine. Ebstein anomaly associated with left ventricular noncompaction. Circulation. 2008;118:e662-4.

3. Thani KB, Khadivi B, Kahn AM, Cotter B, Blanchard D. Ebstein's anomaly with left ventricular noncompaction and bicuspid aortic valve. J Am Coll Cardiol. 2010; 56:899. 
4. Paterick TE, Umland MM, Jan MF, Ammar KA, Kramer C, Khandheria BK, et al. Left ventricular noncompaction: a 25-year odyssey. J Am Soc Echocardiogr. 2012; 25:363-75.
5. Postma AV, van Engelen K, van de Meerakker J, Rahman T, Probst S, Baars MJ, et al. Mutations in the sarcomere gene MYH7 in Ebstein anomaly. Circ Cardiovasc Genet. 2011;4:43-50.

\title{
Right ventricular failure from severe pulmonary hypertension after surgery for shone complex: Back to fetal physiology with reducting, atrioseptectomy, and bilateral pulmonary arterial banding
}

\author{
Heiner Latus, MD, Can Yerebakan, MD, Dietmar Schranz, MD, and Hakan Akintuerk, MD, Giessen, \\ Germany
}

Deciding whether to create a univentricular or biventricular circulation in patients with borderline left ventricular (LV) structures in the neonatal period is challenging. We describe a case in which we used a unique approach intended to imitate cardiac physiology in a "fetal parallel circulation." We call this procedure in neonates with borderline LV the "Giessen hybrid approach." 1

\section{CLINICAL SUMMARY}

A 15-month-old male patient was referred to our center for evaluation of a potential cardiac transplant. Initial diagnostic evaluation and subsequent surgical treatment with neonatal repair of a coarctation of the aorta had been performed in another center for Shone complex, including mitral valve stenosis, LV outflow tract obstruction, stenotic bicuspid aortic valve, and a hypoplastic aortic arch with severe aortic coarctation. Because of multiple life-threatening pulmonary hypertensive crises with right heart failure, the patient underwent reoperation at the age of 9 months. Reoperation included reconstruction of the stenotic aortic valve and relief of the LV outflow tract obstruction, followed by extracorporeal membrane oxygenation treatment and early reoperation for severe mitral regurgitation. Despite specific medical therapy with sildenafil citrate (INN sildenafil), the patient was still affected by $\mathrm{PH}$ with a severe precapillary component,

From the Pediatric Heart Center Giessen, Justus-Liebig University, Giessen, Germany.

Disclosures: Authors have nothing to disclose with regard to commercial support. H.L. and C.Y. contributed equally to the article.

Received for publication April 25, 2014; revisions received June 16, 2014; accepted for publication July 7, 2014; available ahead of print Aug 28, 2014.

Address for reprints: Dietmar Schranz, MD, Pediatric Heart Center, Justus-LiebigUniversity of Giessen, Feulgenstrasse 12, D-35392 Giessen, Germany (E-mail:

dietmar.schranz@paediat.med.uni-giessen.de).

J Thorac Cardiovasc Surg 2014;148:e226-8

$0022-5223 / \$ 36.00$

Copyright (C) 2014 by The American Association for Thoracic Surgery

http://dx.doi.org/10.1016/j.jtcvs.2014.07.042 resulting in suprasystemic pulmonary arterial pressure of $89 / 52 / 67 \mathrm{~mm} \mathrm{Hg}$ and systemic arterial pressure of 69/ $37 / 50 \mathrm{~mm} \mathrm{Hg}$ (pulmonary capillary wedge pressure of $15 \mathrm{~mm} \mathrm{Hg}$ ) and leading to recurrent $\mathrm{PH}$ crises and episodes of cardiopulmonary resuscitation.

At the patient's presentation in our center, echocardiography showed a hypertrophied right ventricle (RV) and a secondary tricuspid regurgitation with a systolic gradient of $100 \mathrm{~mm} \mathrm{Hg}$ (tricuspid valve annulus of $20 \mathrm{~mm}$; $z$ score of 0.7). The LV was small (LV internal dimension in diastole of $22 \mathrm{~mm}$; $z$ score of -2 ), and the mean diastolic gradient across the only minimal regurgitant mitral valve (annulus of $10 \mathrm{~mm} ; z$ score of -3 ) was $8 \mathrm{~mm} \mathrm{Hg}$ (maximal value of $16 \mathrm{~mm} \mathrm{Hg}$ ). The echogenic interventricular septum deviated to the left, the LV outflow tract was narrowed to $5 \mathrm{~mm}$, and the dysplastic and bicuspid aortic valve (annulus of $6 \mathrm{~mm} ; z$ score of -5 ) still had a maximal gradient of $60 \mathrm{~mm} \mathrm{Hg}$.

In light of the patient's medical history with multiple lifethreatening events, the abnormal morphology of the LV with endocardial fibroelastosis (Figure 1), and the large difference in the diameter of the pulmonary and aortic annulus (12 $\mathrm{mm}$ vs $6 \mathrm{~mm}$ ), we decided against a Ross-Konno operation and cardiac transplant and instead opted for a palliative strategy. On the basis of our experience in neonates with a borderline $\mathrm{LV},{ }^{1}$ an 8 -mm polytetrafluoroethylene prosthesis was positioned between the main pulmonary artery and the distal aortic arch, thereby creating a "new arterial duct." The operation was performed through a median sternotomy with cardiopulmonary bypass at $30^{\circ} \mathrm{C}$. This strategy of a "reverse" aortopulmonary shunt was combined with atrial septectomy and bilateral pulmonary arterial banding (bPAB) with a 5-mm polytetrafluoroethylene graft. The patient could be discharged home on postoperative day 21 under medical treatment with bosentan and sildenafil.

After an uneventful period of 4 months without any PH crises, the patient was seen in improved clinical condition, 